\title{
STATE OF THE ART OF CATHETER-DIRECTED THROMBOLYSIS FOR ILIOFEMORAL VENOUS THROMBOSIS
}

\author{
Niels Baekgaard \\ Gentofte Hospital, Copenhagen, Denmark
}

\begin{abstract}
This review is aimed at presenting basic knowledge on catheter-directed thrombolysis (CDT), including stenting and other additional procedures, but without going too far into the fine technical details. The most important published evidence on CDT has been presented, even if many questions associated with this issue still remain unanswered. CDT for iliofemoral venous thrombosis has been known for 25 years. CDT has developed after not very successful attempts to manage iliofemoral thrombosis with systemic or regional thrombolysis, which was associated with mortalities, severe bleedings, and poor recanalisation rates. Administration of a lytic agent directly into the thrombus allows high local concentration of an active substance and minimises systemic action of the drug. Clinical experience has shown that it is obvious that in a case of deep vein thrombosis all occluded veins should be recanalised, with special focus at iliofemoral veins, since the majority of cases of severe post-thrombotic syndrome are associated with poorly recanalised iliac veins. It is also important to implant a stent in the case of residual obstruction of the iliac vein at the same session. A number of factors play a role in successful final outcome, and published guidelines may influence decision making. In order to facilitate sharing the knowledge in this field, it is recommended to use internationally accepted definitions and terms.
\end{abstract}

Key words: iliofemoral deep venous thrombosis, catheter-directed thrombolysis, post-thrombotic syndrome.
REVIEW PAPER

Phlebological Review 2016; 24, 1: 1-6

DOl: $10.5114 /$ pr.2016.61532

Submitted: 5.04 .2016

Accepted: 10.06 .2016

\section{ADDRESS FOR CORRESPONDENCE \\ Dr. Niels Baekgaard \\ Gentofte Hospital \\ Kildegårdsvej 28, DK-2900 Hellerup \\ Copenhagen \\ Denmark \\ e-mail: baekgaard@dadlnet.dk}

emal: baekgard@dadlnet.dk

\section{INTRODUCTION}

Catheter-directed thrombolysis (CDT) is a minimally invasive procedure aimed at local lysis of the thrombus. While using CDT, lytic agent can be administered directly into the thrombus, with controlled dosage of the drug. It offers a number of advantages over systemic thrombolysis. Lytic agents are less concentrated outside the thrombus, thus minimising the risk of fatal bleeding. Also, recanalisation rates are higher with the use of CDT. If needed, it is possible to combine thrombolysis with stent implantation. The first report on CDT in venous system was published in 1991 [1], some years after similar reports on the use of CDT in the arteries. This first case report described the treatment of a young female patient presenting with iliofemoral deep venous thrombosis (DVT); CDT with urokinase followed by balloon angioplasty of residual stenosis of the iliac vein were used in this patient. CDT is included as a recommended treatment in several international guidelines [2, 3]. Nevertheless, published evidence on CDT is quite discordant regarding inclusion criteria, the technique of the procedure, as well as final outcomes.

Many factors may play a role in obtaining a good result of CDT. This review paper is aimed at presenting the most optimal approach to this challenging treatment modality. The terminology and some recommendations are based on a recent update of "Quality improvement guidelines for treatment of lower-extremity deep vein thrombosis with use of endovascular thrombus removal" [4].

\section{MAY-THURNER SYNDROME}

It is well known that compression of the iliac veins, especially on the left side, is a common phenomenon $[5,6]$. Compression of the left common iliac vein (May-Thurner or Cockett syndrome) seems to be of particular importance regarding technical aspects of CDT for iliofemoral DVT. This syndrome is caused by the right common iliac artery that runs above the vein and can compress it against the fifth lumbar vertebra, which in turn may result in stenosis or occlusion, with development of collateral venous network. Such a compression is suspected to play a role in the pathogenesis of iliofemoral DVT with a descending pattern of thrombosis. However, computed tomographic venography reveals compression of iliac veins with at least $25 \%$ lumen reduction, primarily at the left side, in $66 \%$ of "normal" female subjects [7]. On the other hand, patients with left-sided iliofemoral DVT 
present with compression of the iliac veins in $74 \%$ of cases, while only $28 \%$ of controls demonstrated a similar compression (this difference was statistically significant; $p<0.05)$ [8]. It is believed that stenosis of the iliac vein develops as a consequence of repetitive arterial pulsations, finally leading to perivenous fibrosis and development of webs and fibrous annuli inside the vein. However, almost every artery crossing a vein in the pelvic area can produce a compression, but only left-sided iliofemoral DVT is clearly more prevalent in comparison with DVT on the right side (at least three-fold more frequent). In addition, iliofemoral DVT gives poor spontaneous recanalisation (only $30 \%$ of cases), whereas as many as $70 \%$ of femoral DVT will recanalise $[9,10]$. This is of particular importance regarding the risk of development and severity of post-thrombotic syndrome.

\section{INCLUSION AND EXCLUSION CRITERIA OF CDT FOR ILIOFEMORAL DVT}

Catheter-directed thrombolysis for iliofemoral DVT can be performed even in elderly patients because the drug is delivered in high concentration only locally: inside the thrombus. Of course, a patient should be able to cooperate with medical professionals during the treatment and should be able to stay in bed for some days. There are no standard contraindications for teenage patients or for patients of old age $[11,12]$.

Another important issue is the duration of symptoms prior to hospital admission. It seems that irreversible vein wall changes occur after 14 days of DVT [13]. This time limit is also recommended in the guidelines $[2,3]$. On the other hand, the treatment should not be performed in patients who recently underwent surgery or delivery. In such a case, CDT can be performed at least seven days after the event, and until thrombolysis is begun the patient should be given anticoagulation with a weightadjusted dose of a low-molecular-weight heparin. CDT is not recommended in pregnant women as a routine procedure; nonetheless, a few successful procedures have been reported $[14,15]$.
Contraindications for CDT comprise: active cancer, cerebral diseases, liver and kidney insufficiency, uncontrolled hypertension, and bleeding disorders. Previous ipsilateral DVT may represent a relative contraindication, but the clinical decision in such a case depends on the anatomical location of the thrombus. Available vascular access that allows introduction of guidewires and catheters distally from the thrombus constitutes the technical inclusion criterion. The most important inclusion and exclusion criteria are presented in Table 1.

\section{THE LYSIS PROCESS}

Recombinant tissue plasminogen activator (rt-PA) is the most frequently utilised lytic agent. This drug is characterised by a high specificity to plasminogen-bounded fibrin and a half-life of 3-5 minutes. There is about $90 \%$ of the first-pass elimination in the liver of rt-PA, which is in contrast to urokinase, which is characterised by a four-fold longer half-life, and that is why rt-PA is the preferred drug for CDT. This advantage of rt-PA is obvious in a case of major bleeding (intracranial bleeding or bleeding severe enough to result in death, surgery, cessation of therapy, or blood transfusion), when discontinuation of the drug infusion results in complete elimination of the already administered agent within few minutes [4]. The recommended dose of rt-PA for CDT in the veins is $1-2 \mathrm{mg} / \mathrm{h}$, which has been adapted from experience on arterial thrombosis [16]. In our centre maximally $30 \mathrm{mg}$ of $\mathrm{rt}-\mathrm{PA}$ is given, in the CaVenT study half of this daily dose was administered, while maximal total dose of rt-PA established in another trial was $35 \mathrm{mg}[11,17,18]$. The drug is administered into the thrombus through a multi-side-hole catheter with tip occlusion, often using the pulse-spray technique. Heparin administration is an indispensable part of the treatment; otherwise a revascularised vein would reocclude. Unfractionated heparin is either administered directly into the thrombus together with rt-PA, or it is injected via the introducer sheath. The dose of unfractionated heparin should be adjusted according to activat-

Table 1. Inclusion and exclusion criteria, and preprocedural laboratory tests

\begin{tabular}{lcc}
\hline Inclusion criteria & Exclusion criteria & Pre-treatment blood samples \\
\hline DVT involving iliofemoral segment & Cerebral disease & INR \\
History of DVT not longer than 2 weeks & Previous ipsilateral DVT & APTT \\
& Uncontrolled hypertension & Haemoglobin \\
& Hepatic insufficiency & Fibrinogen \\
& Renal insufficiency & Creatinine \\
& Bleeding disorders & Platelet count \\
& INR $>2.0$ & Antithrombin \\
& Antiplatelet treatment & Thrombophilia test \\
& A history of surgery or delivery during last 7 days & Pregnancy test \\
& Active cancer & \\
& Pregnancy and lactation & \\
\hline
\end{tabular}


ed partial thromboplastin time (1.5-2 times more than normal value, maximally 90 seconds), taking into account individual risk of bleeding. If low-molecular-weight heparin is given, its dose should be adjusted according to the patient's weight, and it should be either administered subcutaneously or together with rt-PA. The latter seems to be a safer option, and such a protocol is currently used in our centre. The total volume of lytic infusion (rt-PA and heparin diluted in physiologic saline) should be $3000 \mathrm{ml}$ per day [11].

Access through the popliteal vein using a micropuncture technique under ultrasound guidance is preferred. Coexisting thrombosis of the popliteal vein does not exclude the use of this access. In one paper it has been demonstrated that $90 \%$ of patients presenting with iliofemoral DVT coexisting with popliteal thrombosis had patent popliteal veins after CDT of iliofemoral thrombosis, irrespective of the use of popliteal access [19].

\section{INTERMITTENT PNEUMATIC COMPRESSION}

It is mandatory to enhance venous flow during CDT for venous thrombosis. The rationale for the use of intermittent pneumatic compression (IPC) has been demonstrated by a small Japanese comparative study. In this trial 10 patients were managed without IPC, and in 14 patients IPC (in a sequential manner, under the pressure of 80 $\mathrm{mmHg}$, with inflation of foot cuff for 1-2 seconds, and then calf cuff for 6 seconds, followed by deflation of both cuffs for 60 seconds) was used in addition to CDT. Early recanalisation rates were significantly higher if IPC was added to the therapy $(p=0.015)$, and venous disability scores at follow up 14-22 months after the treatment were also significantly $(p=0.01)$ better. The only criticism associated with this study regarded the fact that patients who were not managed with IPC had significantly shorter duration of symptoms compared to the IPC group (2.9 days vs. 11.8 days; $p=0.007)$. Thus, there might be a bias related to the study design. On the other hand, in this study the dose of urokinase administered to patients presenting with a longer history of DVT was significantly $(p<0.01)$ lower [20]. Some animal studies demonstrated pronounced vasodilatation associated with rapid inflation of IPC cuffs, probably caused by a shear stress-induced release of nitric oxide, which may explain the beneficial effects of this procedure [21]. Nonetheless, the use of IPC is not mentioned in the above-cited recommendation [4]. In a comprehensive review on CDT, which examined papers published from 1998 , only three of 15 publications reported the use of IPC [22]. In our centre IPC remains a routine component of CDT for venous thrombosis [11]. We believe that IPC together with the above-mentioned high-volume infusion of lytic fluid meaningfully enhances the flow in the thrombosed vein.

\section{THROMBUS CLEARANCE, STENTING, AND IVC FILTER}

Strict criteria for thrombus removal, as well as such criteria for stenting, are still missing. Many papers describe thrombus removal in terms of postprocedural ratio of free lumen, according to pre- and post-treatment venograms. A 50\% threshold of thrombus removal is usually reported, still without specific information on the level of the occlusion [4]. The amount of residual thrombus is strongly associated with the efficacy of the lytic solution, duration of symptoms, and duration of the treatment. With a worldwide trend to shorten the treatment time as much as possible, this remaining thrombus material can be a crucial factor associated with unsuccessful outcome. Prolonged CDT increases the rate of complete clot lysis. The decision about when to stop CDT and implant the stent can be based on pressure measurements along the obstruction or on intravascular ultrasound (IVUS). In one study IVUS was used in 67 patients, and the median residual thrombus score revealed by IVUS was significantly higher $(p=0.03)$ if compared to venograms [23]. It has been emphasised that the presence of residual thrombus and lack of patency after six months are strong predictors for the development of post-thrombotic syndrome [24-26]. Therefore, presentations of results of the treatment resulting in patent veins and undamaged valve, given in Kaplan-Meier plots, are still justified.

Stenting of iliac veins is also an area of debate. Successful CDT should result in patent vein on a venogram, spontaneous outflow of injected contrast, and disappearance of collaterals. A lack of such a venographic pattern is suggestive of persistent iliac obstruction and appears to be an inclusion criterion for stenting [27]. Rates of iliofemoral DVT managed with stent implantation substantially differ between the centres. In the review published in 1998, only 6 out of 15 papers reported stenting in $32 \%(83 / 263)$ of patients [21]. In a large multicentre US registry paper published in 1999 a similar rate of stenting was described [28]. Not surprisingly, patency after one year was significantly better in the stenting group compared to the non-stenting patients ( $74 \%$ vs. 53\%; $p=0.001)$. Currently, there is still a divergence regarding stenting rate. In the CaVenT study the stenting rate was $38 \%$, while in our centre this rate is $55 \%[11,17]$.

A routine implantation of IVC filter during CDT is not recommended [4]. This recommendation is based on clinical evidence because no symptomatic pulmonary embolism has been reported in three large studies with almost 300 patients managed and only eight filters implanted $[11,17,29]$.

\section{BIOCHEMICAL MONITORING}

Little has been written regarding this issue. Nonetheless, some parameters can be useful concerning the 
risk of bleeding and the level of clot burden. Evaluation of haemoglobin, fibrinogen, and D-dimer performed daily is still of great value. Decreasing the fibrinogen concentration, which results from its destruction by free-circulating plasmin, is a common phenomenon and accompanies recanalisation of the vein. Similarly, there may be a drop in haemoglobin level. Still, no published studies have specifically examined variations of these laboratory parameters during CDT, looking instead for clinical signs of bleeding. The most frequent source of bleeding is the access site, and haematuria is also quite

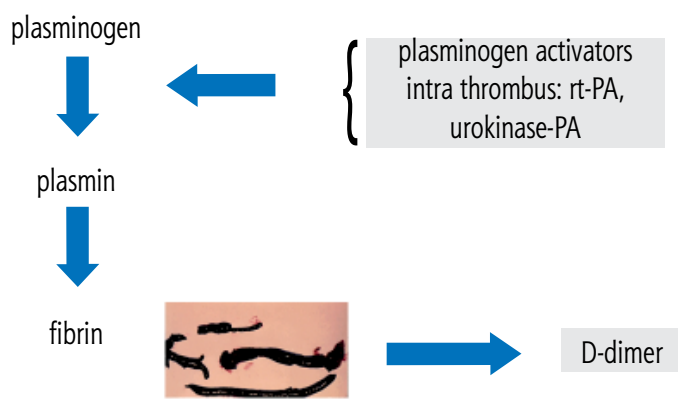

Fig. 1. Elimination of the thrombus under action of rt-PA or urokinase to D-dimer

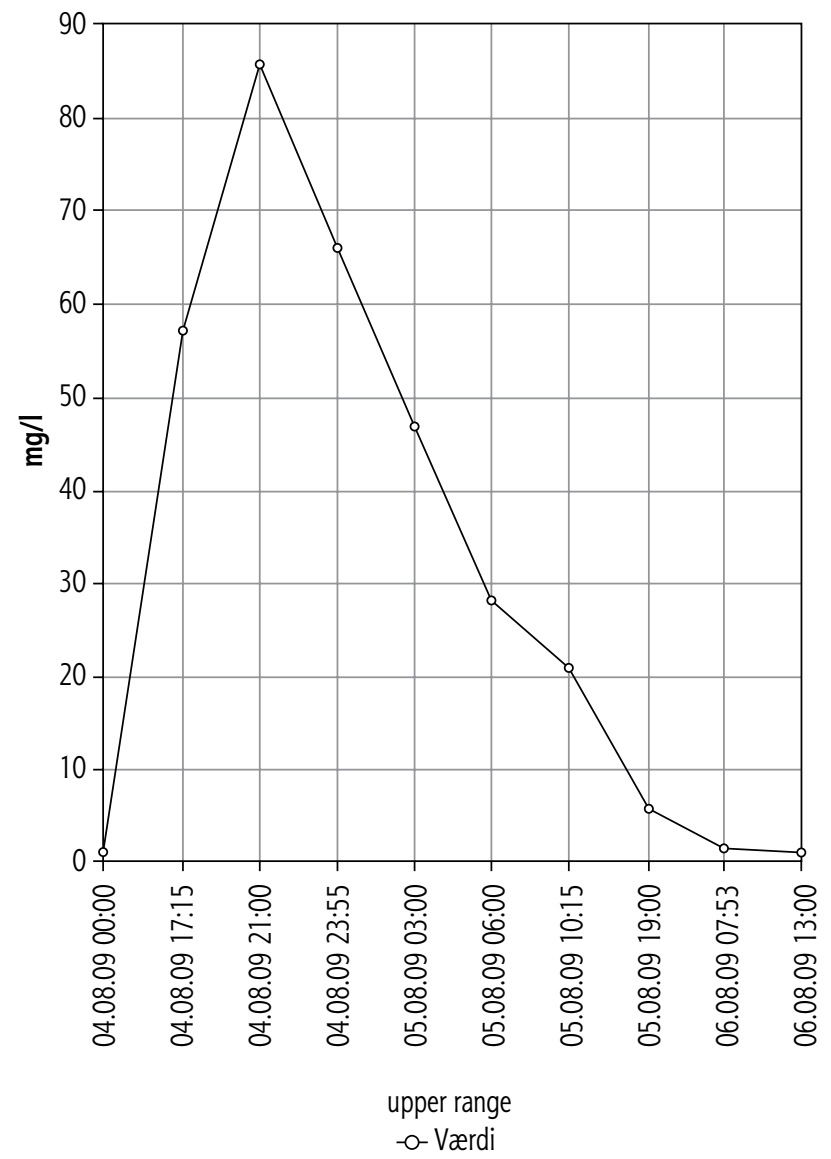

Fig. 2. A typical increase and decrease of D-dimer level during 2 days of treatment with CDT for acute iliofemoral DVT (courtesy of Maja Jørgensen, MD) often seen; these are defined as minor bleeding events. D-dimer is a fibrin degradation product. Successful thrombolysis will increase this blood parameter, and the decreasing level of D-dimer that is seen at the end of treatment is the sign of clot disappearance (Fig. 1). The younger the clot, the more pronounced will be these variations (Fig. 2). In contrast, if only slight variations of D-dimer levels are observed, the thrombus is probably old. In our centre these D-dimer variations are used to monitor clot destruction, and sometimes, if D-dimer is still elevated, we continue infusion of rt-PA for six hours, even if biplane-venograms seem to be normal.

Only one small, recently-published study has addressed the problem of $\mathrm{D}$-dimer concentrations during CDT. In this trial, which evaluated results of CDT in 24 patients, D-dimer concentrations more than $18.4 \mu \mathrm{g} / \mathrm{ml}$ after 12 hours of treatment had a high predictive rate of more than $50 \%$ of lysis [30]. The conclusion drawn from this report is important, but investigation of D-dimer performed at the end of treatment, especially if CDT has been prolonged, seems to be useful as well.

\section{RESULTS OF THE TRIALS}

There have been no fatalities nor pulmonary embolism reported by large trials that have been published during the last five years $[11,17,29]$. Major bleeding was seen in $1-2 \%$ of patients. Information on minor bleedings varies, depending on the accuracy of observations. In our centre there was a $26 \%$ minor bleeding rate, and this included even a tiny oozing to the dressing at the access site, which is of no practical clinical relevance [31]. Reporting the clinical outcome of CDT, including information of a fully recanalised vein with undamaged valves, is of a great importance, especially taking into account the deleterious action of residual thrombi. A publication from our centre reported that in a group of 103 extremities with iliofemoral DVT, after six years $82 \%$ of the veins remained patent and with competent valves. In another group of 109 patients managed in our centre, after nine years post-thrombotic syndrome developed in $16.5 \%$ of them $[11,32]$. A large Norwegian CaVenT randomised trial (90 vs. 99 patients) demonstrated that at two-year follow-up the absolute risk reduction of post-thrombotic syndrome was $14.4 \%$ in a group managed with CDT compared to a group of patients who received only anticoagulation [17]. Five-year follow-up of these patients revealed absolute risk reduction at the level of $28 \%$, but no benefit in terms of quality of life was found [33]. Nevertheless, the results of this trial should be interpreted with caution, since only every second patient presented with iliac DVT. It is possible that the level of risk reduction would be even higher if only iliac DVT were included, since in this location CDT is clearly superior to anticoagulation alone. A meta-analysis published in 2012, based on four studies on CDT 
vs. anticoagulation alone, concluded that there is a significant increase in patency of the vein if CDT is used (risk reduction: 0.38; 95\% CI: 0.18-0.37) and significant risk reduction of post-thrombotic syndrome (risk reduction: 0.19; 95\% CI: 0.07-0.48) [34]. Another review, summarising the results of three randomised controlled trials and three comparative studies, confirmed that CDT was more effective than anticoagulation alone [35].

\section{CONCLUSIONS}

Catheter-directed thrombolysis for the management of acute iliofemoral DVT is a justified treatment modality. It has been demonstrated that with such a treatment the risk of post-thrombotic syndrome can be reduced. There is a lot of discrepancy regarding technical details of CDT in recently published papers. In this review I tried to emphasise the clinical value of some measures, such as: high dose of rt-PA (no less than $1 \mathrm{mg} / \mathrm{h}$ ), simultaneous administration of heparin, utilisation of IPC, stenting of any residual obstruction of the iliac vein, possible benefit from D-dimer monitoring for thrombus disappearance, and monitoring of fibrinogen aimed at decreasing the risk of bleeding. Besides, it is very important to follow inclusion and exclusion criteria for CDT. Although elderly and very young patients can be managed with CDT, unrestricted patient's cooperation is a must. As yet, there is no evidence of any beneficial effect of compression therapy with medical stockings. Also, no clinical benefit of life-long anticoagulation, except for patients with severe thrombophilia or atresia of the inferior vena cava, has been demonstrated. At the moment we are awaiting the results of the American ATTRACT and Dutch CAVA studies. Hopefully, these trials will elucidate some uncertainties surrounding endovascular treatment for iliofemoral DVT.

It should also be remembered that all statements and recommendations given in this review paper should be taken into account if CDT is augmented by mechanical thrombectomy or other techniques aimed at shortening the duration of treatment [36]. Besides, safety of CDT is very important. Some suggestions how to optimise, simplify, and standardise the monitoring of thrombolysis have been recently published [37].

The author declares no conflict of interest.

\section{References}

1. Okrent D., Messersmith R., Buckman J. Transcatheter fibrinolytic therapy and angioplasty for left iliofemoral venous thrombosis. J Vasc Interv Radiol 1991; 2: 195-197.

2. Meissner M.H., Gloviczki P., Comerota A.J., Dalsing M.C., Eklof B.G., Gillespie D.L., Lohr J.M., McLafferty R.B., Murad M.H., Padberg F., Pappas P., Raffetto J.D., Wakefield T.W.; Society for Vascular Surgery; American Venous Forum. Early thrombus removal strategies for acute deep venous thrombosis:
Clinical practice guidelines of the Society for Vascular Surgery and the American Venous Forum. J Vasc Surg 2012; 55: 14491462 .

3. Kahn S.R., Comerota A.J., Cushman M., Evans N.S., Ginsberg J.S., Goldenberg N.A., Gupta D.K., Prandoni P., Vedantham S., Walsh M.E., Weitz J.I.; American Heart Association Council on Peripheral Vascular Disease, Council on Clinical Cardiology, and Council on Cardiovascular and Stroke Nursing. The postthrombotic syndrome: evidence-based prevention, diagnosis, and treatment strategies: a scientific statement from the American Heart Association. Circulation 2014; 130: 1636-1661.

4. Vedantham S., Sista A.K., Klein S.J., Nayak L., Razavi M.K., Kalva S.P., Saad W.E., Dariushnia S.R., Caplin D.M., Chao C.P., Ganguli S., Walker T.G., Nikolic B.; Society of Interventional Radiology and Cardiovascular and Interventional Radiological Society of Europe Standards of Practice Committees. Quality improvement guidelines for treatment of lower-extremity deep vein thrombosis with use of endovascular thrombus removal. J Vasc Interv Radiol 2014; 25: 1317-1325.

5. May R., Thurner J. The cause of the predominantly sinistral occurrence of thrombosis of the pelvic veins. Angiology 1957; 8: 419-427.

6. Cockett F.B., Thomas M.L., Negus D. Iliac vein compression: its relation to iliofemoral thrombosis and the postthrombotic syndrome. Br Med J 1967; 2: 14-19.

7. Kibbe M.R., Ujiki M., Goodwin A.L., Eskandari M., Yao J., Matsumura J. Iliac vein compression in an asymptomatic patient population. J Vasc Surg 2004; 39: 937-943.

8. Oguzkurt L., Ozkan U., Ulusan S., Koc Z., Tercan F. Compression of the left common iliac vein in asymptomatic subjects and patients with left iliofemoral deep vein thrombosis. J Vasc Interv Radiol 2008; 19: 366-370.

9. Akesson H., Brudin L., Dahlström J.A., Eklöf B., Ohlin P., Plate G. Venous function assessed during a 5 year period after acute ilio-femoral venous thrombosis treated with anticoagulation. Eur J Vasc Surg 1990; 4: 43-48.

10. Caprini J.A., Arcelus J.I., Hoffman K.N., Size G., Laubach M., Traverso C.I., Coats R., Finke N., Reyna J.J. Venous duplex imaging follow-up of acute symptomatic deep vein thrombosis of the leg. J Vasc Surg 1995; 21: 472-476.

11. Bækgaard N., Broholm R., Just S., Jørgensen M., Jensen L.P. Long-term results using catheter-directed thrombolysis in 103 lower limbs with acute iliofemoral venous thrombosis. Eur J Vasc Endovasc Surg 2010; 39: 112-117.

12. Engelberger R.P., Fahrni J., Willenberg T., Baumann F., Spirk D., Diehm N., Do D.-D., Baumgartner I., Kucher N. Fixed low-dose ultrasound-assisted catheter-directed thrombolysis followed by routine stenting of residual stenosis for acute ilio-femoral deepvein thrombosis. Thromb Haemost 2014; 111: 1153-1160.

13. Bækgaard N., Foegh P., Wittens C.H., Arnoldussen C. Thrombus age is ideally measured by history or MRV prior to thrombus removal. Phlebology 2015; 30 (Suppl 1): 20-26.

14. Holly B.P., Werner E., DeStephano C., Lessne M.L. Single-session, pharmacomechanical thrombetomy of deep venous thrombosis in the pregnant patient. J Vasc Interv Radiol 2014; 25: 1308-1311.

15. Herrera S., Comerota A.J., Thakur S., Sunderji S., DiSalle R., Kazanjian S. N., Assi Z. Managing iliofemoral deep venous thrombosis of pregnancy with a strategy of thrombus removal is safe and avoids post-thrombotic morbidity. J Vasc Surg 2014; 59: 456-464. 
16. Vearhaghe H., Stockx L., Lacroix H., Vermylen J., Baert A.L. Catheter-directed thrombolysis of iliofemoral vein thrombosis with use of rt-PA. Eur Radiol 1997; 7: 996-1001.

17. Enden T., Haig Y., Kløw N.E., Slagsvold C.-E., Sandvik L., Hafsdal H., Holme P.A., Holmen L.O., Njaatad A.M., Sandbæk G., Sandset P.M. Long-term outcome after additional catheter-directed thrombolysis versus standard treatment for acute iliofemoral deep venous thrombosis (the CaVenT study): a randomised controlled trial. Lancet 2012; 379: 31-38.

18. Comerota A.J. The ATTRACT trial: rationale for early intervention for iliofemoral DVT. Perspect Vasc Surg Endovasc Ther 2009; 21: 221-224.

19. Jeyabalan G., Marone L., Rhee R., Hirsch S., Makaroum M.S., Cho J., Chaer R.A. Inflow thrombosis does not adversely affect thrombolysis outcomes of symptomatic iliofemoral deep vein thrombosis. J Vasc Surg 2011; 54: 448-453.

20. Ogawa T., Hoshino S., Midorikawa H., Sato K. Intermittent pneumatic compression of the foot and calf improves the outcome of catheter-directed thrombolysis using low-dose urokinase in patients with acute proximal venous thrombosis of the leg. J Vasc Surg 2005; 42: 940-944.

21. Liu K., Chen L.E., Seaber A.V., Urbaniak J.R. Influence of rate and duration on vasodilatory effect by intermittent pneumatic compression in distant skeletal muscle. J Orthop Res 1999; 17: 415-420.

22. Grossman C., McPherson S. Safety and efficacy of catheter-directed thrombolysis for iliofemoral venous thrombosis. Am J Roentgenol 1999; 172: 667-672.

23. Raju S., Davis M., Martin A. Assessment of residual thrombus after venous thrombolytic regimes. J Vasc Surg Venous Lymphat Dis 2014; 2: 148-154.

24. Comerota A.J., Grewal N., Martinez J.T., Chen J.T., DiSalle R., Andrews L., Sepanski D., Assi Z. Postthrombotic morbidity correlates with residual thrombus following catheter-directed thrombolysis for iliofemoral deep vein thrombosis. J Vasc Surg 2012; 55: 768-773.

25. Haig Y., Enden T., Slagsvold C.E., Sandvik L., Sandset P.M., Kløw N.E. Residual rates of reflux and obstruction and their correlation to post-thrombotic syndrome in a randomized study on catheter-directed thrombolysis for deep venous thrombosis. J Vasc Surg Venous Lymphat Disord 2014; 2: 123-130.

26. Avgerinos E.D., Hager E.S., Naddaf A., Dillavou E., Singh M., Chaer R.A. Outcomes and predictors of failure of thrombolysis for iliofemoral deep venous thrombosis. J Vasc Surg Venous Lymphat Disord 2015; 3: 35-41.

27. Bækgaard N., Just S., Foegh P. Which criteria demand additive stenting during catheter-directed thrombolysis. Phlebology 2014; 29 (1S): 112-118.

28. Mewissen M.W., Seabrook G.R., Meissner M.H., Cynamon J., Labropoulos N., Haughton S.H. Catheter-directed thrombolysis for lower extremity deep venous thrombosis: report of a national multicenter registry. Radiology 1999; 211: 39-49.

29. Protack C.D., Bakken A.M., Patel N., Saad W.E., Waldman D.L., Davies M.G. Long-term outcomes of catheter directly thrombolysis for lower extremity deep venous thrombosis without prophylactic inferior vena cava filter placement. J Vasc Surg 2007; 45: 992-997.

30. Luo C.M., Wu I.H., Chan C.Y., Chen Y.S., Yang W.S., Wang S.S. Dimerized plasmin fragment $\mathrm{D}$ as a potential biomarker to predict successful catheter-directed thrombolysis therapy in acute deep vein thrombosis. Phlebology 2015; 30: 620-626.
31. Bækgaard N, Klitfod L, Broholm R. Safety and efficacy of catheter-directed thrombolysis. Phlebology 2012; Suppl 1: 149-154.

32. Broholm R., Sillesen H., Damsgaard M.T., Jørgensen M., Just S., Jensen L.P., Bækgaard N. Postthrombotic syndrome and quality of life in patients with iliofemoral venous thrombosis treated with catheter-directed thrombolysis. J Vasc Surg 2011; 54 (Suppl 6): 18S-25S.

33. Haig Y., Enden T., Grøtta O., Kløw N.E., Slagsvold C.E., Ghanima W., Sandvik L., Hafsahl G., Holme P.A., Holmen L.O., Njaastad A., Sandbæk G., Sandset P.M. Post-thrombotic syndrome after catheter-directed thrombolysis for deep vein thrombosis (CaVenT): 5-year follow-up results of an open-label, randomized controlled trial. Lancet Haemotol 2016; 3: 64-71.

34. Casey E.T., Murad M.H., Zumaeta-Garcia M., Elamin M.B., Shi Q., Erwin P.J., Montori V.M., Gloviczki P., Meissner M. Treatment of acute iliofemoral deep venous thrombosis. J Vasc Surg 2012; 55: 1463-1473.

35. Du G.C., Zhang M.C., Zhao I.C. Catheter-directed thrombolysis plus anticoagulation versus anticoagulation alone in treatment of proximal thrombosis-a meta-analysis. Vasa 2015; 44: 195-202.

36. Malgor R.D., Gasparis A.P. Pharmaco-mechanical thrombectomy for early thrombus removal. Phlebology 2012; 27 Suppl 1: 155-162.

37. Bækgaard N., Klitfod L., Jørgensen M. Should catheter-directed thrombolysis be monitored? Phlebology 2016; 31 (1S): 5-10. 